\title{
Utilizing dendritic scaffold for feasible formation of naphthalene excimer
}

\author{
P K LEKHA, TUFAN GHOSH and EDAMANA PRASAD* \\ Department of Chemistry, Indian Institute of Technology Madras, Chennai 600036, India \\ e-mail: pre@iitm.ac.in
}

\begin{abstract}
Peripheral functionalization of PAMAM dendrimers with naphthalene units leads to significant ground state aggregation in the system above the critical aggregation concentrations (CAC). Upon photoexcitation of the ground state aggregates, static type excimer formation of naphthalene moiety is observed. Significant red-shifted emission from naphthalene excimers is achieved through generating the static type excimers in polar solvents such as methanol and acetonitrile-water mixtures. Control experiments suggest that the presence of dendritic scaffold in the system play a pivotal role in generating intense static excimer emission in naphthalene modified PAMAM dendrimers, in solution phase at room temperature.
\end{abstract}

Keywords. PAMAM dendrimer; dendrimer aggregation; static excimer; naphthalene excimer.

\section{Introduction}

Dendrimers are functional macromolecules with threedimensional fractal geometry which makes them potentially suitable candidates for various applications in fields such as energy harvesting, nano-catalysis and delivery of drugs as well as other biologically important molecules to living cells. ${ }^{1}$ One of the unique aspects of dendrimers is that the physicochemical properties of these macromolecules can be altered by attaching suitable functional groups at the periphery of the structure. ${ }^{2}$ In this regard, peripheral modification of dendrimers is of paramount interest in current research as it is the most convenient strategy for designing suitable dendritic structures for specific applications. ${ }^{3}$

When the dendrimer periphery is modified by fluorophore units, it is expected that a fraction of the fluorophores can form excimer upon photo-excitation, due to the close proximity of the fluorophore units at the periphery of the dendrimer. While such 'intra-molecular' excimers based on dendrimer substitution are known, ${ }^{4,5}$ 'inter-molecular' excimer formation based on peripheral modification of dendrimers are rarely reported in the literature. Recent results from our laboratory suggest that incorporation of anthracene and pyrene moieties to the periphery of poly(amidoamine) \{PAMAM dendrimers results in the formation of 'inter-molecular' excimer formation. ${ }^{6}$ This type of excimer formation was mainly assisted by the solvent induced aggregation of

\footnotetext{
${ }^{*}$ For correspondence
}

the peripherally modified dendrimers, where the fluorophore units are forced to stay close to each other, prior to the photo-excitation process. It was noticed that the dendritic scaffold of the PAMAM dendrimer plays a pivotal role in paving the way for excimer formation. In this work, we are extending the above mentioned approach to generate naphthalene excimer, in order to verify the universal nature of the strategy for feasible excimer formation through PAMAM dendrimer aggregation.

There are only a few reports of naphthalene functionalized dendrimers in literature. Vögtle et al. synthesized the naphthalene modified dendrimers with a cyclam core where the luminescence from naphthalene was used to detect various metal ions. ${ }^{7,8}$ Ming Li et al. synthesized naphthalene cored poly(aryl ether) dendron and studied the intermolecular exciplex formation as a result of singlet electron transfer process from dendritic backbone to the naphthyl group in dichloromethane. They observed that back folding conformation of the dendrimers leads to the feasible formation of the exciplex. ${ }^{9}$ A few reports in the literature describes the energy and charge transfer in naphthalene functionalized dendrimers and the associated photophysical properties of the systems. ${ }^{9,10}$ Nonetheless, investigations on the effect of dendritic scaffolds on the feasibility of naphthalene excimer formation has not been reported in the literature.

Here, we report synthesis and photophysical study of naphthalene functionalized zero and first generation PAMAM dendrimers in order to understand the mechanism of excimer formation in the system. Results from the experiments suggest that peripherally modified 


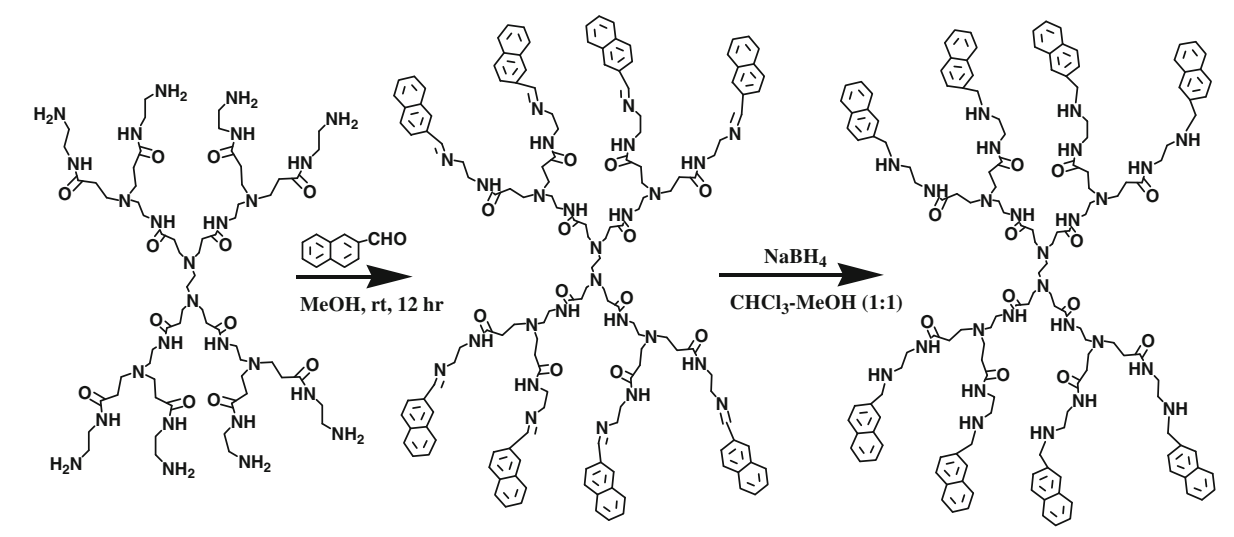

Scheme 1. Synthesis of naphthalene functionalized first generation PAMAM dendrimers. Detailed synthetic procedure is given in the supporting information.

PAMAM dendrimers with naphthalene significantly aggregate in the ground state, above their critical aggregation concentration (CAC). As a result of the aggregation, pre-arranged naphthalene dimers are formed in the ground state, which readily form 'static' type excimer upon photo-excitation. ${ }^{11,12}$ Reports regarding the static excimer emission from naphthalene in solution phase are quite rare in the literature. The static excimer emission from crystalline naphthalene has been reported. ${ }^{11}$ For example, Uchida and co-workers observed excimer formation in naphthalene microcrystals obtained by grinding single crystals of naphthalene at liquid nitrogen temperatures. ${ }^{11 \text { a }}$ Excimer emission from crystalline naphthalene was also achieved at elevated pressure conditions. ${ }^{11 \mathrm{~b}}$ Naphthalene excimers formed in solution state was effectively utilized for the detection of metal ions. ${ }^{12}$ However, static type excimer emission from naphthalene modified PAMAM dendrimers is seldom reported in the literature and the results presented here suggest that the strategy of utilizing dendritic scaffold for generating naphthalene excimer is a highly feasible process. More importantly, our results show that the proper selection of solvent can result in significantly red-shifted excimer emission from naphthalene modified PAMAM dendrimers.

\section{Experimental}

\subsection{Instruments}

UV-Vis experiments were performed on a Jasco V 660 UV-Vis spectrophotometer. Luminescence experiments were carried out on a Horiba Jobin Yvon Fluoromax4 fluorescence spectrophotometer. The path length of optical cell used was $1 \mathrm{~cm}$. and the slit width for exci- tation and emission spectra was $2 \mathrm{~nm}$. The fluorescence decay measurements were carried out by the time correlated single-photon counting technique (TCSPC) with a microchannel plate photomultiplier tube (MCPPMT) as detector and picosecond laser as excitation source (model $5000 \mathrm{U}, \mathrm{IBH}, \mathrm{UK}$ ). All emission spectra reported are corrected emission spectra.

\subsection{Synthesis of naphthalene functionalized PAMAM dendrimers}

The naphthalene modified poly(amidoamine) \{PAMAM dendrimers (compounds II and III) were synthesized by condensation of 2-napthalenecarboxaldehyde and amine terminated zero, and first generations PAMAM dendrimers in alcoholic medium. ${ }^{13}$ The corresponding amine terminated PAMAM-naphthalene conjugates (compounds $\mathbf{V}$ and $\mathbf{V I}$ ) were synthesized by the in situ reduction of imine precursors using sodium borohydride. ${ }^{14}$ Scheme 1 shows the synthetic procedure for the synthesis of naphthalene functionalized PAMAM dendrimers. The naphthalene functionalized PAMAM dendrimers are represented by PAMAM- $(\mathrm{Np})_{\mathrm{n}}$ where $n$ is the number of naphthalene units attached per dendrimer. Naphthalene substituted ethylene diamine (compound I) was used as a model compound for control experiment.

\section{Results and discussion}

The structure of the compounds in the present study is shown in chart 1 . The synthetic scheme utilized to prepare the naphthalene functionalized PAMAM dendrimer is given in scheme 1. 


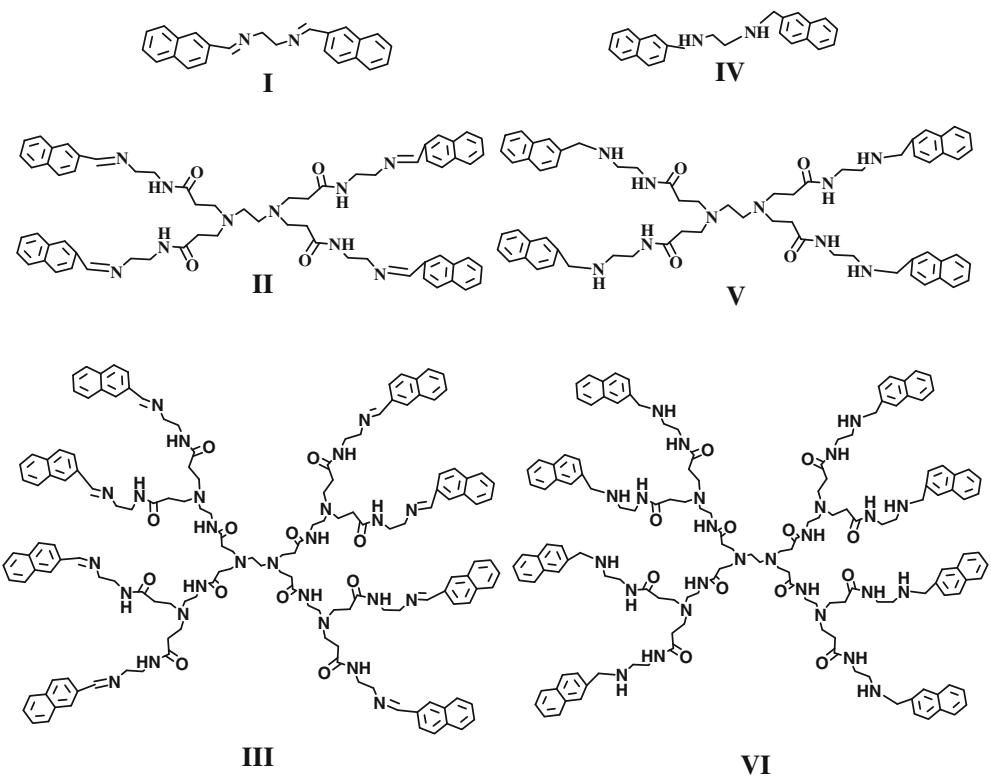

Chart 1. Compounds used for the present study.

\section{UV-Vis absorption spectra of naphthalene functionalized PAMAM dendrimer}

UV-Visible absorption spectra of the compounds I to III were recorded in dichloromethane. All compounds exhibited structured absorption bands corresponding to naphthalene monomer at concentrations below $10^{-6} \mathrm{M}$. However, significant changes were observed in the absorption spectrum at higher concentrations (i.e., above $10^{-5} \mathrm{M}$ ). Figure 1 shows the UV-Vis absorption spectra of compound III in dichloromethane at lower and higher concentrations regime. Similar observations were found with other compounds also (see Supporting material figures $\mathrm{S} 1$ and $\mathrm{S} 2$ ).

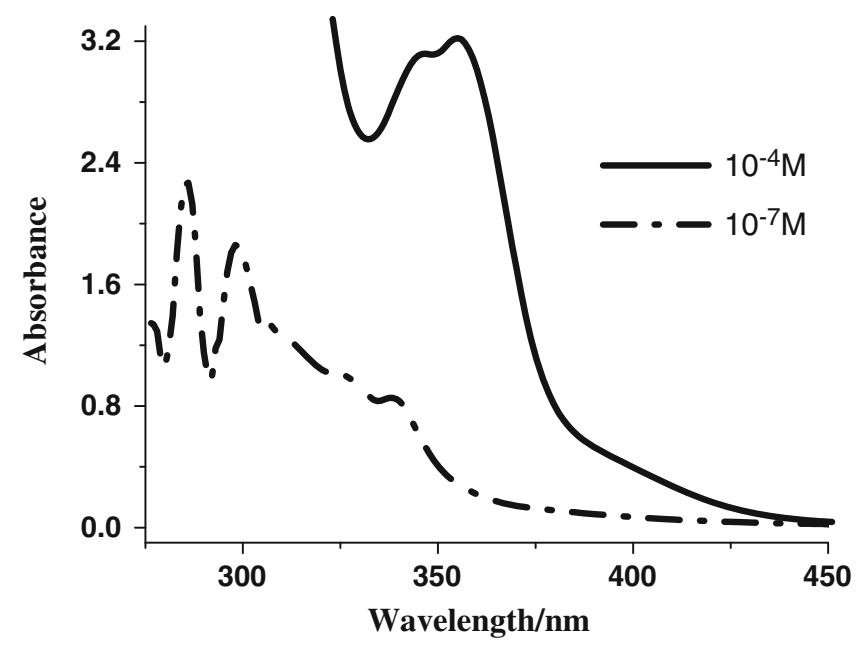

Figure 1. UV-Vis absorption spectra of compound III in dichloromethane at lower and higher concentrations.
The structured absorption bands of compound III appeared around 275, 285, 298, 325 and $334 \mathrm{~nm}$ in dichloromethane at lower concentration $\left(10^{-7} \mathrm{M}\right)$. At concentrations higher than $10^{-5} \mathrm{M}$, a new shoulder band appeared around $360 \mathrm{~nm}$. Similar behaviour was noticed for compounds I and II in dichloromethane as well as solvents such as chloroform and methanol. We attribute the new shoulder band absorption to the ground state naphthalene dimer formation. Strikingly similar observations were noticed previously, for anthracene and pyrene functionalized PAMAM dendrimers. ${ }^{6}$

\subsection{Absorption studies of compounds IV-VI in dichloromethane}

The UV-Vis absorption spectra of compounds IV to VI were checked in dichloromethane and similar results were obtained. In this case, the appearance of shoulder band was visible at relatively high concentrations $\left(>10^{-4} \mathrm{M}\right)$. Figure 2 shows comparison spectra of compounds III and VI in dichloromethane.

In order to verify whether the shoulder band absorption is originated from the charge transfer complexes between amine moieties in the PAMAM dendrimer and naphthalene units, the UV-Vis spectrum of all the compounds shown in chart 1 was examined in the presence of acid (1 equivalent to the number of nitrogen atoms present in the dendrimer) where the nitrogen atoms are protonated and charge-transfer probability can be neglected. Surprisingly, the absorption band $\sim 360 \mathrm{~nm}$ appears with identical intensity to that in the absence 


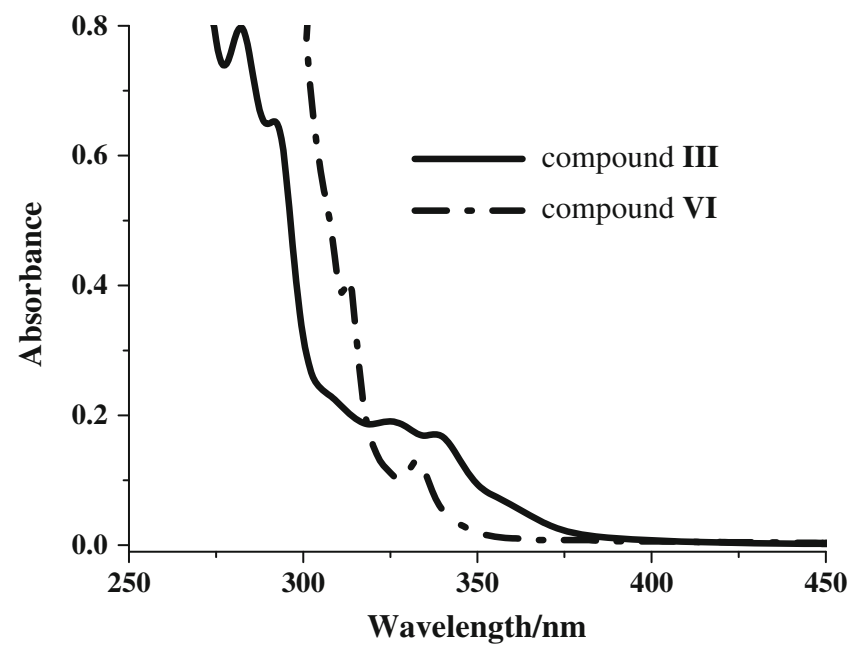

Figure 2. UV-Vis absorption spectra of compounds III and VI in dichloromethane, concentration of the sample in both cases were $10^{-5} \mathrm{M}$.

of a proton source, which rule out the charge transfer possibility in the present case (figure S3). ${ }^{15}$

\subsection{Critical aggregation concentration of naphthalene functionalized PAMAM dendrimers}

The UV-Vis absorption studies of naphthalene modified PAMAM dendrimers were carried out over a wide range of concentrations $\left(10^{-3} \mathrm{M}\right.$ to $\left.10^{-7} \mathrm{M}\right)$. The results suggest that these dendrimers aggregate in solution above a critical aggregation concentration (CAC). Interestingly, a concentration independent behaviour was observed in the case of model compounds I and IV.

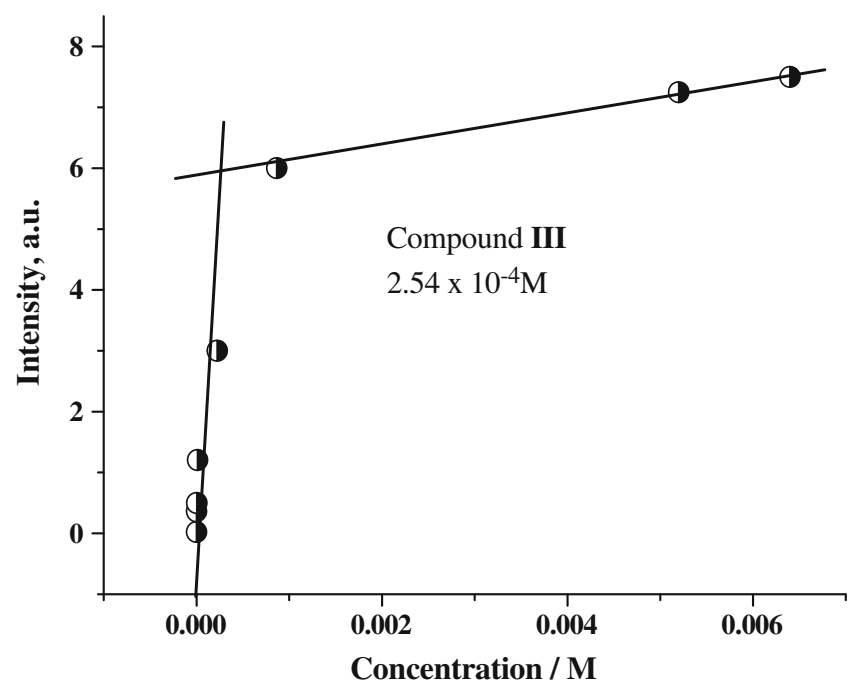

Figure 3. A plot of fluorescence intensity $v s$ concentration of compound III in acetonitrile:water $(20 \% \mathrm{v} / \mathrm{v})$. The CAC was determined from the point of intersection in the plot.
This demonstrates the important role of utilizing the dendritic scaffolds in the present study. The plot of the excimer emission intensity $v s$ substrate concentration of PAMAM- $(\mathrm{Np})_{\mathrm{n}}$ dendrimers shows clear inflection point, from which the CAC were determined. The values were found to be $3.06 \times 10^{-4} \mathrm{M}$ and $2.54 \times 10^{-4} \mathrm{M}$ for PAMAM- $(\mathrm{Np})_{4}$ and PAMAM- $(\mathrm{Np})_{8}$ dendrimers, respectively with 5\% error margin. Figure 3 shows the $\mathrm{CAC}$ plot for PAMAM- $(\mathrm{Np})_{8}$ dendrimers.

\section{Steady state fluorescence properties of PAMAM-(Np) $)_{n}$ aggregates}

Steady state fluorescence properties from the system have been investigated in dichloromethane. Upon exciting compounds I to III $\left(10^{-5} \mathrm{M}\right)$ at $280 \mathrm{~nm}$, naphthalene monomer emission was observed between 300 and $375 \mathrm{~nm} .{ }^{16-18}$ However, photoexcitation at the shoulder band absorption resulted in a strong structureless and broad emission of naphthalene excimer centred at $440 \mathrm{~nm}$. Figure 4 contains the emission spectrum of PAMAM- $(\mathrm{Np})_{8}$ dendrimer at two different excitation wavelengths.

The excimer emission from the system was then monitored in a series of solvents such as toluene, ethyl acetate, chloroform, ethanol, methanol acetonitrile and acetonitrile-water mixtures $(20 \% \mathrm{v} / \mathrm{v})$. In the case of toluene and ethyl acetate, only monomer emission was observed even at relatively higher concentrations $\left(10^{-4} \mathrm{M}\right)$. In the case of chloroform, ethanol and methanol, the excimer emission was reasonably good at concentrations above CAC. A red shift in excimer emission was observed in the case of high dielectric solvents such as methanol and acetonitrile. Photoexcitation of model compound $\mathbf{I}$ in methanol has an intense broad emission centred at $470 \mathrm{~nm}$ (figure S4), where as the emission was further red shifted to $510 \mathrm{~nm}$ in corresponding dendritic systems. Figure 5 shows the steady state fluorescence spectra of compound III in methanol.

The emission was further red shifted to $525 \mathrm{~nm}$ in the case of acetonitrile-water mixture. Figure 6 shows the emission spectra of PAMAM- $(\mathrm{Np})_{8}$ imine dendrimer in acetonitrile with $20 \% \mathrm{v} / \mathrm{v}$ water.

The red-shifted emission spectra from the dendrimer derivatives are likely due to the enhanced stabilization of the excited state by the high solvent dielectric. ${ }^{19}$ It was also noted that excimer formation was more prominent in polar solvents compared to non-polar solvents. This is presumably because of the enhanced hydrophobic force between the naphthalene units under a polar 

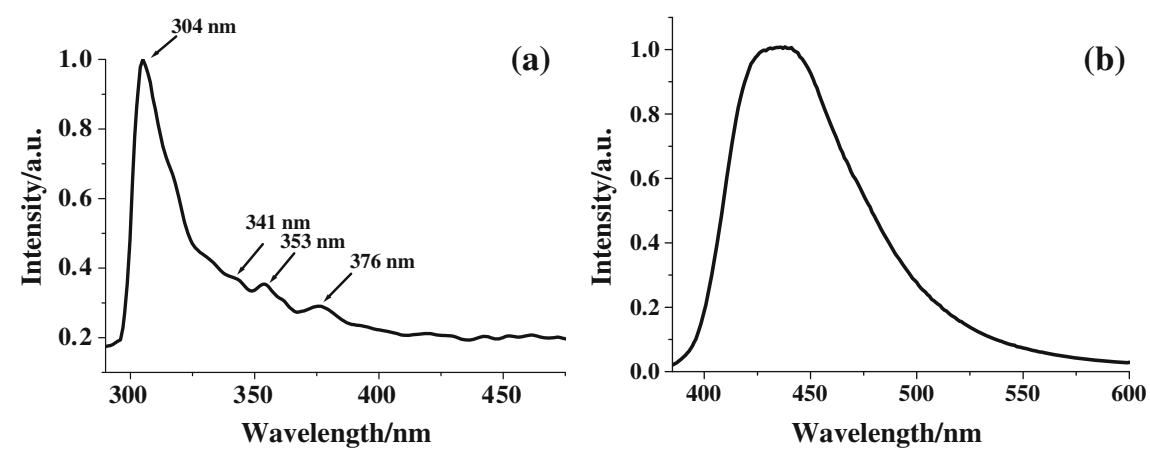

Figure 4. Steady state fluorescence spectrum of compound III in dichloromethane. (a) $\lambda_{\mathrm{ex}}=280 \mathrm{~nm}$ and (b) $\lambda_{\mathrm{ex}}=370 \mathrm{~nm}$, concentration of the sample is $10^{-4} \mathrm{M}$.

environment, which enhances the propensity to aggregate in the ground state. It is interesting to note that not only general solvent effects but specific solvent effect also plays a crucial role in controlling the energetics of the excimer formation. For example, the excimer formation was feasible in chloroform and not in ethyl acetate even though the polarity of ethyl acetate is slightly higher than that of chloroform. This could be due to the specific solvent interactions such as hydrogen bonding between the amide carbonyl groups and the solvent milieu of chloroform, which associate the molecules in the ground state to close proximity.

The compounds IV to VI exhibited completely different type of photophysical properties. The aggregation propensity of these naphthalene dendrimers (compounds IV to VI) were less compared to the corresponding imine precursors (compounds I to III). This may be

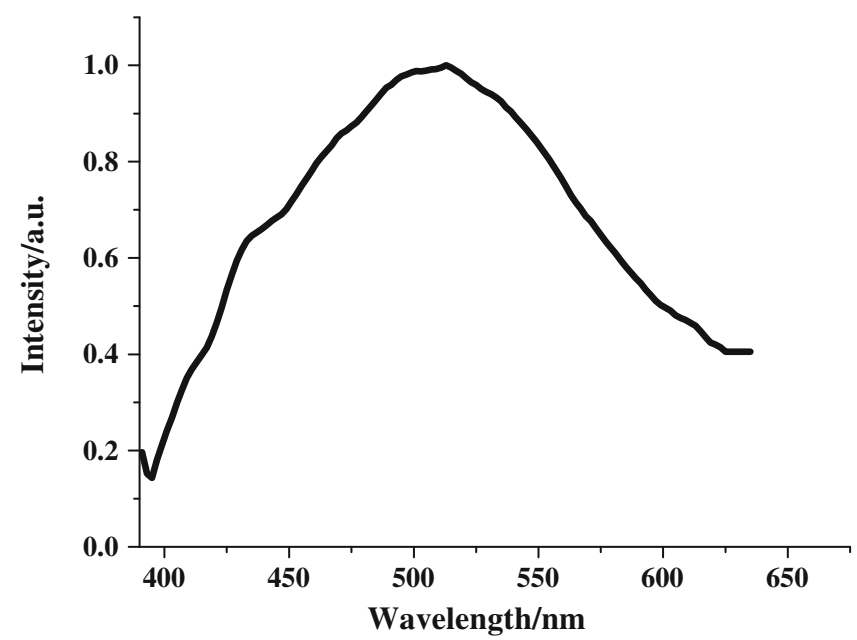

Figure 5. Steady state fluorescence spectrum of compound III in $\mathrm{MeOH}$, concentration $=10^{-4} \mathrm{M}, \lambda_{\mathrm{ex}}=380 \mathrm{~nm}, \lambda_{\mathrm{em}}=$ $510 \mathrm{~nm}$. attributed to the lack of conjugation in them, which affects the planarity of the naphthalene units in the individual dendrimer, leading to unfavourable geometry for excimer formation. The fluorescence emission quantum yields of naphthalene functionalized dendrimers in methanol, dichloromethane and acetonitrile-water mixture were calculated using coumarine and fluorescein as reference compounds and the values are given in table $1 .{ }^{20}$

The relatively high quantum yields of PAMAM$(\mathrm{Np})_{\mathrm{n}}$ where $\mathrm{Np}$ units are attached through imine bonds suggest that the propensity of aggregation is more in compounds II and III, compared to that in compounds $\mathbf{V}$ and VI. Thus, excimer formation was more feasible with compounds I to III.

Excimers can be classified into dynamic and static type according to the mechanism through which they

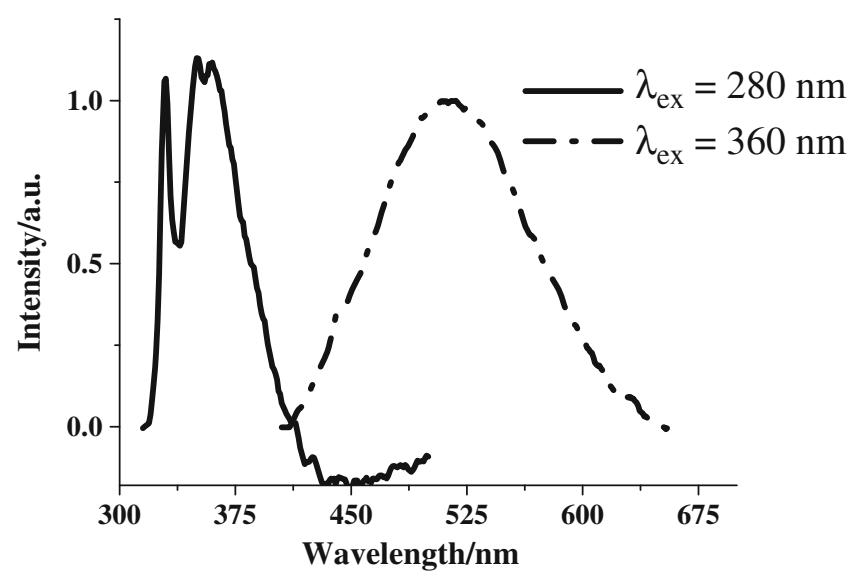

Figure 6. Steady state fluorescence spectra of compound III in ACN with $20 \% \mathrm{v} / \mathrm{v}$ water mixture at two different excitation wavelengths. Concentration of the sample is $10^{-4} \mathrm{M}$. 
Table 1. Fluorescence emission quantum yields of static excimers of compound I to VI in various solvents.

\begin{tabular}{lccc}
\hline Compound & Methanol & $\mathrm{CH}_{2} \mathrm{Cl}_{2}$ & ACN- $\mathrm{H}_{2} \mathrm{O}$ mixture \\
\hline I & 0.09 & 0.11 & 0.06 \\
II & 0.12 & 0.13 & 0.10 \\
III & 0.32 & 0.25 & 0.17 \\
IV & 0.05 & 0.02 & 0.03 \\
V & 0.09 & 0.04 & 0.07 \\
VI & 0.11 & 0.12 & 0.10 \\
\hline
\end{tabular}

error $= \pm 5 \%$

are generated. Scheme 2 describes the schematic representation of the formation of different types of excimers.

The dynamic excimers are formed as a result of the diffusion of a ground state fluorophore towards an excited state fluorophore, during the lifetime of the excited state species. Whereas, the static excimers are formed as a result of the photoexcitation of a pre-existing ground state complex. In such cases, the monomer units are arranged in such a way that the distance between them will be close enough to form the excimer readily, upon photo-excitation. Typically, the critical distance between two monomer units to form static type excimer is 3.5-3.9 $\AA$, in the case of poly cyclic hydrocarbons. ${ }^{21}$

The pre-arranged fluorophores can be generated in the ground state by properly selecting the solvent milieu. It is known from the literature that, poly cyclic aromatic hydrocarbons tend to aggregate in the ground state in polar medium due to the hydrophobic forces between the non-polar groups. ${ }^{6}$ Such solvent-induced aggregates render an opportunity to generate static excimers, through the mechanism described above. This can be verified by recording the fluorescence excitation spectra of the sample, where the emission is collected at both monomer and excimer emission maxima. Significant red shifted emission is expected in the excitation spectrum collected at the excimer emission

$$
\begin{aligned}
\mathbf{M} \stackrel{\mathbf{h} v}{\longrightarrow} \mathbf{M}^{*} \\
\mathbf{M}^{*}+\mathbf{M} \stackrel{\text { Diffusion }}{\longrightarrow} \mathbf{E}_{\mathbf{D}}^{*}[\text { Dynamic excimers }]
\end{aligned}
$$$$
\mathbf{M}---\mathbf{M} \longrightarrow \mathbf{h} \boldsymbol{\longrightarrow} \mathbf{E}_{\mathbf{S}}{ }^{*}[\text { Static excimers] }
$$

Pre arranged

monomer units

Scheme 2. Mechanism of dynamic and static type excimer formation.

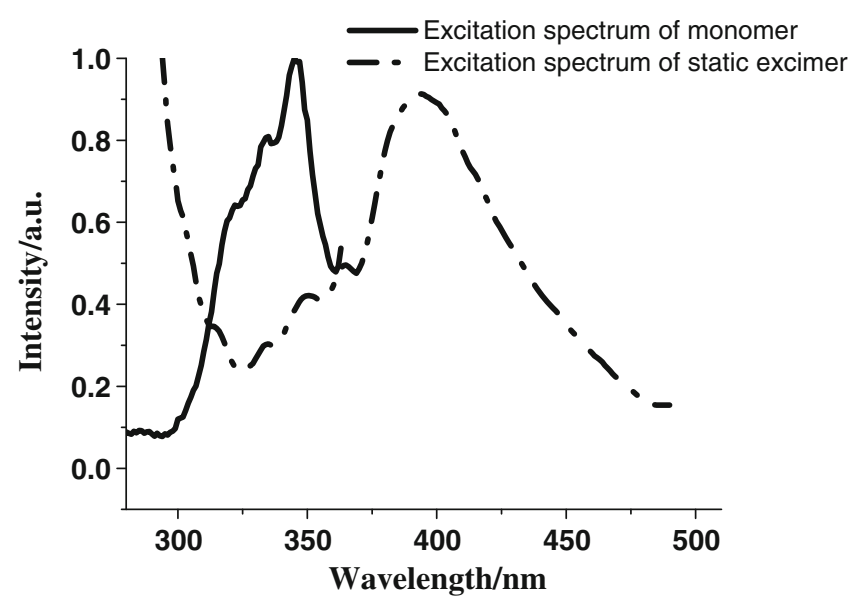

Figure 7. Excitation spectra of III collected from monomer at $350 \mathrm{~nm}$ (solid) and from excimer at $525 \mathrm{~nm}$ (dash-dot) in $\mathrm{ACN}-$ Water $(20 \% \mathrm{v} / \mathrm{v})$ mixture. $[\mathbf{I I I}]=10^{-4} \mathrm{M}$.

maximum, if the excimer emission is static in nature. Figure 7 shows the excitation spectra of PAMAM $(\mathrm{Np})_{8}$ imine dendrimer in ACN - Water (20\% v/v) mixture, where the emission is collected at the monomer and excimer emission maxima. The excitation spectrum of the excimer emission from compound III in $\mathrm{ACN}$ Water $(20 \mathrm{v} / \mathrm{v})$ mixture was red shifted by $55 \mathrm{~nm}$ corresponding to that of the monomer, clearly indicating the formation of a static excimer in the present case. ${ }^{22}$

\section{Time resolved fluorescence studies}

In order to understand the excited state lifetime features of static type excimers formed in the system, time correlated single photon counting experiments were carried out. The compounds were excited at $370 \mathrm{~nm}$ using a LED source, and the emission from the system was monitored at $435 \mathrm{~nm}$ in dichloromethane and $510 \mathrm{~nm}$ in methanol. Upon exciting the system at $370 \mathrm{~nm}$, a double exponential decay was observed in both solvents (figure 8). The multi components in the decay can be originated from two sources: one is due to the formation of a small fraction of dynamic excimer since monomer naphthalene has an absorption tail at the excitation wavelength. The other option is that different types of naphthalene excimers can be formed as a result of the formation of different types of ground state aggregates of naphthalene units, where the geometry of the individual fluorophore units can vary widely. The lifetime values were $0.79 \mathrm{~ns}$ and $4.45 \mathrm{~ns}$ in dichloromethane and $1.71 \mathrm{~ns}$ and $3.43 \mathrm{~ns}$ in methanol. Picraux et al. observed dynamic excimer emission from naphthalene appended to a dinuclear iron-oxo complex and the excited state 

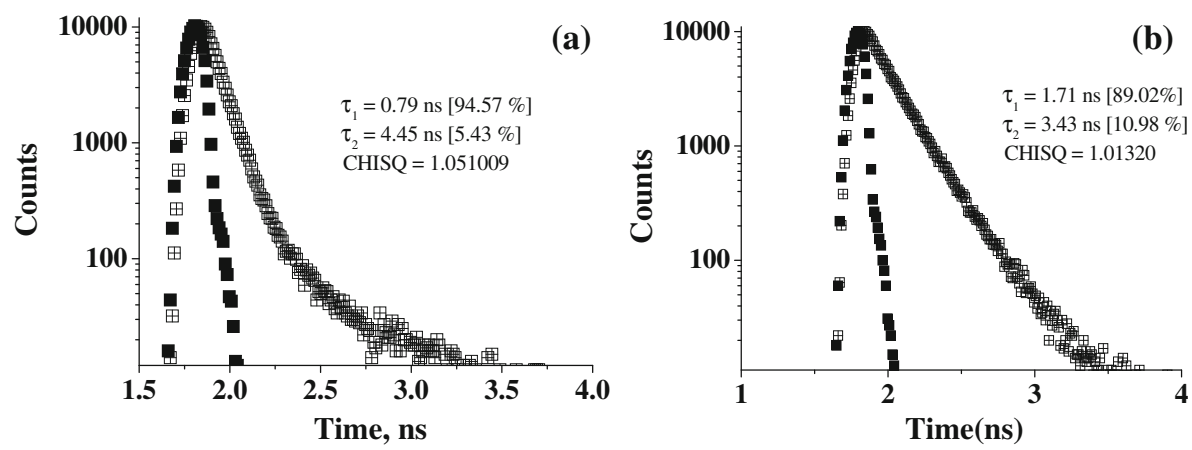

Figure 8. Excited state decay traces of compound III in dichloromethane (a) and in methanol (b). $\lambda_{\text {ex }}$ was $370 \mathrm{~nm}$. Emission collected at $470 \mathrm{~nm}$ for dichloromethane and $510 \mathrm{~nm}$ for methanol. The lifetime data of the remaining compounds in the present study is given in the supporting information (table S1).

lifetime value was $4.6 \mathrm{~ns} .{ }^{23}$ The short lifetime component is, thus, attributed to the static excimer of naphthalene, while the relatively longer lifetime component is attributed to the dynamic excimer emission from naphthalene.

While both type of excimers of naphthalene are formed upon exciting the naphthalene decorated PAMAM dendrimers, the relative amplitude of the excited species clearly indicate that static excimer is formed in large excess, compared to dynamic type. For example, the relative amplitude values of the short lifetime component for compounds I to III are close to $90 \%$ whereas only $10 \%$ of the species corresponding to the long lifetime component was formed. This corroborates our hypothesis that functionalizing dendritic structures by fluorescent moieties can be utilized to generate static excimers, leading to intensely emitting systems with tunable light emitting properties in solution phase, at room temperature.

\section{Conclusion}

In summary, zero and first generation PAMAM dendrimers were peripherally modified by naphthalene moieties and the effect of aggregation of the dendritic structures in the ground state on the propensity of the excimer formation by naphthalene units was examined. The critical aggregation concentrations for the systems were determined and it was observed that the aggregation propensity was high in dendrimers where the fluorophore (naphthalene) is attached to the dendrimer backbone through an imine bond. The relatively high polar nature of the PAMAM- $(\mathrm{Np})_{\mathrm{n}}$ dendrimers resulted in extremely red shifted static excimer emission from naphthalene in polar solvents, upon photo-excitation.

\section{Supporting information}

The electronic supporting information can be seen in www.ias.ac.in/chemsci.

\section{Acknowledgements}

We thank the Department of Science and Technology (DST) (SR/S1/PC-26/2007), Govt. of India for the financial support. We thank Professor A K Mishra, Indian Institute of Technology Madras for time resolved experiments.

\section{References}

1. (a) Medina S H, El-Sayed M E H 2009 Chem. Rev. 109 3141; (b) Yu G S, Bae Y M, Choi H, Kong B, Choi I S, Choi J S 2011 Bioconjug. Chem. 221046

2. Voegtle F, Richard G, Werner N 2009 Dendrimer chemistry; Wiley-VCH, Weinheim, Baden-Württemberg

3. Balzani V, Ceroni P, Maestri M, Vicinelli V 2003 Curr. Opin. Chem. Biol. 7 657; (b) Astruc D, Boisseleir E, Ornelas C 2010 Chem. Rev. 1101857

4. Cicchi S, Fabbrizzi P, Ghini G, Brandi A, Foggi P, Marcelli A, Righini R, Botta C 2009 Chem. Eur. J. 15 754

5. Wang B-B, Zhang X, Jia X-R, Li Z-C, Ji Y, Yang L, Wei Y 2004 J. Am. Chem. Soc. 12615180

6. Lekha P K, Prasad E 2010 Chem. Eur. J. 163699

7. Saudan C, Balzani V, Ceroni P, Gorka M, Maestri M, Vicinelli V, Vögtle F 2003 Tetrahedron 593845

8. Saudan C, Balzani V, Gorka M, Lee S K, Maestri M, Vicinelli V, Vögtle F 2003 J. Am. Chem. Soc. 125 4424

9. Li M, Li Y, Zeng Y, Chen J, Li Y 2009 J. Phys. Chem. C 11311554

10. Hahn U, Gorka M, Vögtle F, Vicinelli V, Ceroni P, Maestri M, Balzani V 2002 Angew. Chem. Int. Ed. Engl. 413595 
11. (a) Uchida K, Tanaka M and Tomura M 1979 J. Lumin. 20 409; (b) Jones P F, Nicol M 1968 J. Chem. Phys. 48 5440; (c) Sturtevant J, Webber S E 1989 Macromolecules 223564

12. Zhang H, Xie L, Yan W, He C, Cao X, Duan C 2009 New. J. Chem. 331478

13. Martinez R, Espinosa A, Tarraga A, Molina P 2005 Org. Lett. 75869

14. James T D, Shinmori H, Takeuchi M, Shinkai S 1996 Chem. Commun. 6705

15. Ramamurthy V, Schanze K S (eds.) 2001 Understanding and manipulating excited state processes; New York: Eastern Hemisphere Distribution

16. Vala Jr T M, Haebig J, Rice S A 1965 J. Chem. Phys. 43 886
17. Kobayashi T, Fukaya T, Fujii N 2000 J. Membr. Sci. 164 157

18. Morishima Y, Lim H S, Nozakura S, Sturtevant J L 1989 Macromolecules 221148

19. Valeur B 2002 Molecular fluorescence-principles and application; Weinheim: Wiley-VCH

20. Lakowicz J R 2006 Principles of fluorescence spectroscopy; New York: Springer

21. Masu H, Mizutani I, Ono Y, Kishikawa K, Azumaya I, Yamaguchi K, Kohmoto S 2006 Cryst. Growth Des. 6 2086

22. Jung H S, Park M, Han D Y, Kim E, Lee C, Ham S, Kim J S 2009 Org. Lett. 113378

23. Picraux L B, Weldon B T, Mc Cusker J K 2003 Inorg. Chem. 42273 